\title{
Observation of a Floquet symmetry-protected topological phase with superconducting qubits
}

Dong-Ling Deng ( $\nabla$ dldeng@umich.edu )

Tsinghua University https://orcid.org/0000-0002-1042-4646

\section{Xu Zhang}

Zhejiang University https://orcid.org/0000-0002-6102-0035

\section{Wenjie Jiang}

Tsinghua University

Jinfeng Deng

Zhejiang University

\section{Ke Wang}

Zhejiang University

Jiachen Chen

Zhejiang University

\section{Pengfei Zhang}

Zhejiang University https://orcid.org/0000-0001-8908-4228

\section{Wenhui Ren}

Zhejiang University

\section{Hang Dong}

Zhejiang University

\section{Xuhao Zhu}

Zhejiang University

\section{Yu Gao}

Zhejiang University

\section{Feitong Jin}

Zhejiang University

\section{Qiujiang Guo}

Zhejiang University https://orcid.org/0000-0003-1093-3405

\section{Hekang Li}

Zhejiang University https://orcid.org/0000-0003-3914-4979

\section{Chao Song}

Department of Physics, Zhejiang University

\section{Alexey Gorshkov}

University of Maryland https://orcid.org/0000-0003-0509-3421

Thomas ladecola 
lowa State University

\section{Fangli Liu}

Joint Quantum Institute, Joint Center for Quantum Information and Computer Science, and Physics

Department, University of Maryland and National Institute of Standards and Technology https://orcid.org/0000-0002-5690-4995

\section{Zhexuan Gong}

University of Maryland

\section{Zhen Wang}

Interdisciplinary Centre for Quantum Information and Zhejiang Province Key Laboratory of Quantum Technology and Device, Department of Physics, Zhejiang University

\section{Haohua Wang}

Zhejiang University https://orcid.org/0000-0001-5380-4297

\section{Physical Sciences - Article}

Keywords: quantum physics, non-equilibrium phases, symmetry-protected topological states

Posted Date: October 28th, 2021

DOI: https://doi.org/10.21203/rs.3.rs-963503/v1

License: (c) (1) This work is licensed under a Creative Commons Attribution 4.0 International License. Read Full License

Version of Record: A version of this preprint was published at Nature on July 20th, 2022. See the published version at https://doi.org/10.1038/s41586-022-04854-3. 


\title{
Observation of a Floquet symmetry-protected topological phase with superconducting qubits
}

\author{
Xu Zhang ${ }^{1, *}$, Wenjie Jiang ${ }^{2, *}$, Jinfeng Deng ${ }^{1, *}$, Ke Wang $^{1}$, Jiachen Chen $^{1}$, Pengfei Zhang ${ }^{1}$, Wenhui Ren ${ }^{1}$, Hang Dong $^{1}$, \\ Shibo $\mathrm{Xu}^{1}$, Yu Gao ${ }^{1}$, Feitong Jin ${ }^{1}$, Xuhao $\mathrm{Zhu}^{1}$, Qiujiang Guo ${ }^{4,3}$, Hekang $\mathrm{Li}^{1,3}$, Chao Song ${ }^{1,3}$, Alexey V. Gorshkov ${ }^{5}$, \\ Thomas Iadecola $^{6,7}$, Fangli Liu ${ }^{5,8}$, Zhe-Xuan Gong ${ }^{9,10}$, Zhen Wang ${ }^{1,3, \dagger}$, Dong-Ling Deng ${ }^{2,11, \ddagger}$, and H. Wang ${ }^{1,3,4}$ \\ ${ }^{1}$ Interdisciplinary Center for Quantum Information, State Key Laboratory of Modern Optical Instrumentation, \\ and Zhejiang Province Key Laboratory of Quantum Technology and Device, \\ Department of Physics, Zhejiang University, Hangzhou 310027, China \\ 2 Center for Quantum Information, IIIS, Tsinghua University, Beijing 100084, China \\ ${ }^{3}$ Alibaba-Zhejiang University Joint Research Institute of Frontier Technologies, Hangzhou 310027, China \\ ${ }^{4}$ Hangzhou Global Scientific and Technological Innovation Center, Zhejiang University, Hangzhou 311215, China \\ ${ }^{5}$ Joint Quantum Institute and Joint Center for Quantum Information and Computer Science, \\ University of Maryland and NIST, College Park, MD, USA \\ ${ }^{6}$ Department of Physics and Astronomy, Iowa State University, Ames, Iowa 50011, USA \\ 7 Ames Laboratory, Ames, Iowa 50011, USA \\ ${ }^{8}$ QuEra Computing Inc., Boston, Massachusetts 02135, USA \\ ${ }^{9}$ Department of Physics, Colorado School of Mines, Golden, Colorado 80401, USA \\ ${ }^{10}$ National Institute of Standards and Technology, Boulder, Colorado 80305, USA \\ ${ }^{11}$ Shanghai Qi Zhi Institute, 41th Floor, AI Tower, No. 701 Yunjin Road, Xuhui District, Shanghai 200232, China
}

\begin{abstract}
Quantum many-body systems away from equilibrium host a rich variety of exotic phenomena that are forbidden by equilibrium thermodynamics. A prominent example is that of discrete time crystals [1-8], where time translational symmetry is spontaneously broken in periodically driven systems. Pioneering experiments have observed signatures of time crystalline phases with trapped ions [9, 10], spins in nitrogen-vacancy centers [11-13], ultracold atoms [14, 15], solid spin ensembles [16, 17], and superconducting qubits [18-20]. Here, we report the observation of a distinct type of intrinsically non-equilibrium state of matter, a Floquet symmetry-protected topological phase, which is implemented through digital quantum simulation with an array of programmable superconducting qubits. Unlike the discrete time crystals reported in previous experiments, where spontaneous breaking of the discrete time translational symmetry occurs for local observables throughout the whole system, the Floquet symmetry-protected topological phase observed in our experiment breaks the time translational symmetry only at the boundaries and has trivial dynamics in the bulk. More concretely, we observe robust long-lived temporal correlations and sub-harmonic temporal response for the edge spins over up to 40 driving cycles using a circuit whose depth exceeds 240. We demonstrate that the sub-harmonic response is independent of whether the initial states are random product states or symmetry-protected topological states, and experimentally map out the phase boundary between the time crystalline and thermal phases. Our work paves the way to exploring novel non-equilibrium phases of matter emerging from the interplay between topology and localization as well as periodic driving, with current noisy intermediate-scale quantum processors [21].
\end{abstract}

Symmetry-protected topological (SPT) phases are characterized by non-trivial edge states that are confined near the boundaries of the system and protected by global symmetries [22-26]. In a clean system without disorder, these edge states typically only occur for the ground states of systems with a bulk energy gap. At finite temperature, they are typically destroyed by mobile thermal excitations. However, adding strong disorder can make the system many-body localized (MBL) [27-31], allowing for a sharply defined topological phase and stable edge states even at infinite temperature [32-36]. Strikingly, the topological phase and corresponding edge states can even survive external periodic driving, as long as the driving frequency is large enough so that the localization persists $[37,38]$.

The interplay between symmetry, topology, localization, and periodic driving gives rise to various peculiar phases of matter that exist only out of equilibrium [38]. Understanding and categorizing these unconventional phases poses a notorious scientific challenge. On the theoretical side, topological classifications of periodically driven (Floquet) systems with
[4, 39-41] and without [42] interactions have already been obtained through a range of mathematical techniques (such as group cohomology), revealing a number of "Floquet SPT" (FSPT) phases with no equilibrium counterparts [38]. Yet, we still lack powerful analytical tools or numerical algorithms to thoroughly address these phases and their transitions to other ones. On the experimental side, signatures of discrete time crystals (DTCs) [1-8], which are paradigmatic examples of exotic phases beyond equilibrium [43], have been reported in a wide range of systems [9-20]. However, none of these experiments encompass topology as a key ingredient. A recent experiment simulating an FSPT phase on a trapped-ion quantum computer found that the phase was short-lived due to the presence of coherent errors in the device [44]. Realizing a long-lived FSPT phase, which demands a delicate concurrence of topology, localization, and periodic driving, thus still remains a notable experimental challenge.

Here, we report the observation of an intrinsically nonequilibrium FSPT phase with a programmable array of superconducting qubits (Fig. 1) with high controllability and 


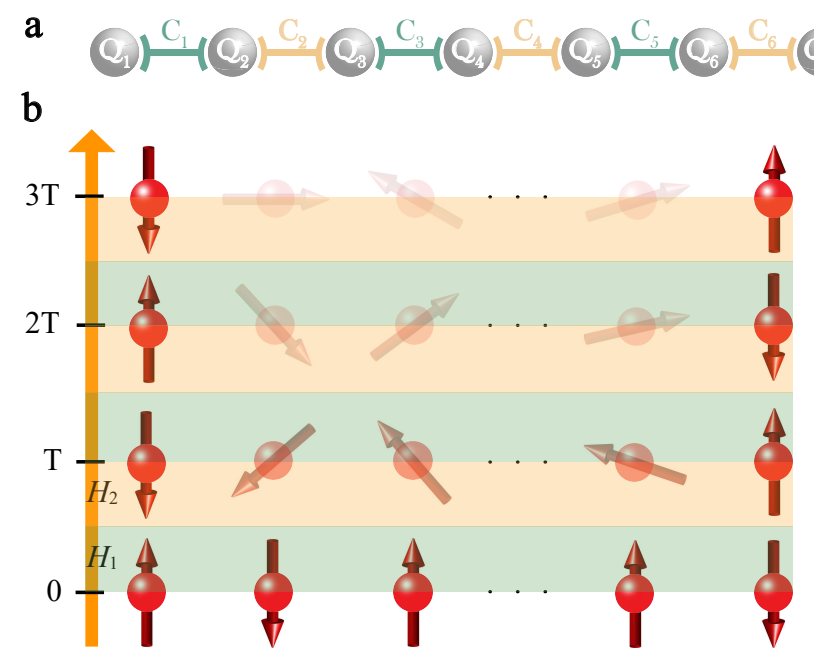

C

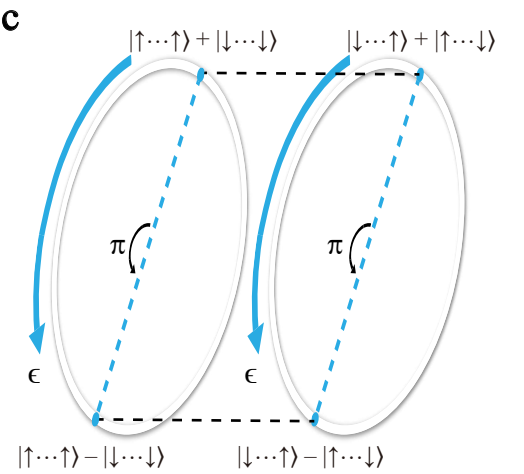

e

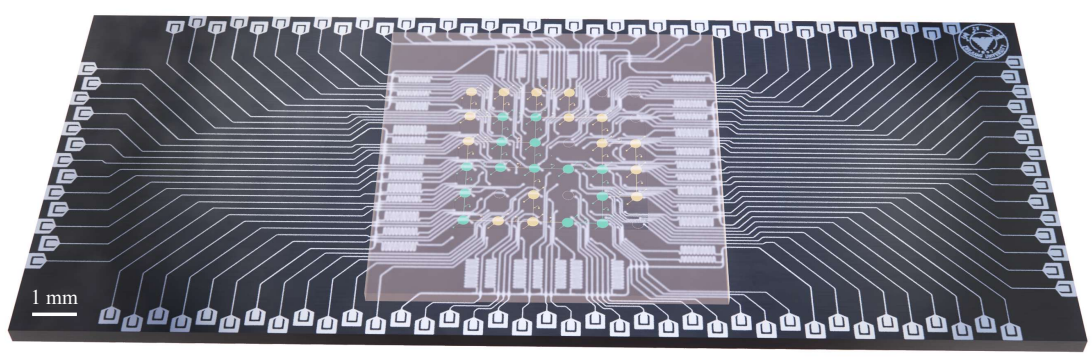

FIG. 1. FSPT phase and schematics of the experimental setup. a, Fourteen qubits used in our experiment are coupled to their neighbors with capacitive couplers. b, A chain of spins is periodically driven with the time-periodic Hamiltonian $H(t)$, giving rise to an FSPT phase characterized by time translational symmetry breaking at the boundaries. c, The quasienergy spectrum $(\epsilon)$ of the Floquet unitary $U_{F}$, which is the time evolution operator over one period. For the FSPT phase, every eigenstate is two-fold degenerate and has a cousin separated by quasienergy $\pi$. Here, $|\uparrow \cdots \uparrow\rangle \pm|\downarrow \cdots \downarrow\rangle$ and $|\downarrow \cdots \uparrow\rangle \pm|\uparrow \cdots \downarrow\rangle$ denote the eigenstates of $U_{F}$, and we use two identitical quasienergy circles to explicitly show the two-fold degeneracy (Sumplementary Information I. B). d, A schematic illustration of the experimental circuits used to implement the time dynamics governed by the time-periodic Hamiltonian $H(t)$. We randomly sample the Hamiltonians and prepare the initial states as random product states or random static SPT states. After running a sequence of quantum gates, we measure the local magnetization or stabilizer operators at discrete time points. e, Illustration of the quantum processor, with the 14 qubits highlighted in green.

long coherence time. We successfully implement the dynamics of the system under a prototypical time-periodic Hamiltonian, which we theoretically predict to exhibit an FSPT phase. This requires us to digitally simulate a three-body-interacting Hamiltonian exhibiting an SPT phase via a large-depth quantum circuit obtained using a novel neuroevolution algorithm [45]. We then measure local spin magnetizations and their temporal correlations and demonstrate that both quantities show a subharmonic response at the boundaries but not in the bulk of the chain. This situation differs drastically from the case of DTCs, which exhibit subharmonic response everywhere in the bulk. This contrast stems from a fundamental distinction between DTC and FSPT phases: the former exhibit conventional long-range order in the bulk intertwined with the spontaneous breaking of discrete time-translational symmetry $[43,46,47]$ while the latter exhibit SPT order that can only be revealed through boundary effects or nonlocal "string oper- ators" in the bulk $[39,41,48]$. The observed boundary subharmonic response persists over an extended range of parameters and is robust to various experimental imperfections, independent of the initial states. We further explore the FSPT phase experimentally from the perspectives of entanglement dynamics, entanglement spectrum, and the dynamics of stabilizer operators that underlies its topological nature. By measuring the variance of the subharmonic peak height in the Fourier spectrum, we also experimentally map out the phase boundary between the time crystalline and thermal phases. This work opens the door to harnessing this exotic phase of matter for practical quantum information processing.

\section{Model Hamiltonian and its implementation}

We consider a one-dimensional spin- $\frac{1}{2}$ chain governed by the 
following time-periodic Hamiltonian (see Fig. 1b):

$$
\begin{aligned}
H(t) & =\left\{\begin{array}{ll}
H_{1}, & \text { for } 0 \leq t<T_{1} \\
H_{2}, & \text { for } T_{1} \leq t<T
\end{array},\right. \\
H_{1} & \equiv\left(\frac{\pi}{2}-\delta\right) \sum_{k} \hat{\sigma}_{k}^{x}, \\
H_{2} & \equiv-\sum_{k}\left[J_{k} \hat{\sigma}_{k-1}^{z} \hat{\sigma}_{k}^{x} \hat{\sigma}_{k+1}^{z}+V_{k} \hat{\sigma}_{k}^{x} \hat{\sigma}_{k+1}^{x}+h_{k} \hat{\sigma}_{k}^{x}\right],
\end{aligned}
$$

where $\hat{\sigma}_{k}^{x, z}$ is the Pauli matrix acting on the $k$-th spin; $J_{k}, V_{k}$, and $h_{k}$ are random parameters drawn independently from uniform distributions over $\left[J-\Delta_{J}, J+\Delta_{J}\right],\left[V-\Delta_{V}, V+\Delta_{V}\right]$, and $\left[h-\Delta_{h}, h+\Delta_{h}\right]$, respectively. For simplicity, we fix $T=2 T_{1}=2$ throughout this paper. We note that $H(t)$ has a $Z_{2} \times Z_{2}$ symmetry. For a suitable parameter regime, it has been shown that $H_{2}$ can be in an MBL phase, where topological edge states can survive as coherent degrees of freedom at arbitrarily high energies [34]. The localization and edge states carry over to the case of periodic driving with the Hamiltonian $H(t)$, giving rise to an FSPT phase. In this FSPT phase, the time translational symmetry only breaks at the boundary but not in the bulk. The Floquet unitary that fully characterizes the FSPT phase reads $U_{F}=U_{2} U_{1}$, where $U_{1}=e^{-i H_{1}}$ and $U_{2}=e^{-i H_{2}}$ are the unitary operators generated by the Hamiltonians $H_{1}$ and $H_{2}$, respectively. The quasienergy specturm of $U_{F}$ reveals that every eigenstate is two-fold degenerate and has a cousin eigenstate separated by quasienergy $\pi$ (see Fig. 1c). The degenerate eigenstates also exhibit longrange mutual information between the boundary spins; this is essential for the robustness of the subharmonic response of the edge spins against local perturbations, including finite $\delta$ and $V_{k}$, that respect the protecting $\mathbb{Z}_{2} \times \mathbb{Z}_{2}$ symmetry. We refer to the Methods and Supplementary Information for a more in-depth discussion about the model.

To implement $H(t)$ with superconducting qubits, the threebody term in $H_{2}$, which is crucial for the SPT phase at high energy, poses an apparent challenge since no three-body interaction appears naturally in the superconducting system. We thus employ the idea of digital quantum simulation [49] to implement $H(t)$ with quantum circuits (Fig. 1d). For $V_{k}=h_{k}=0$, we find optimal circuits in an analytical fashion that can implement $H(t)$ with arbitrary $J_{k}$ and $\delta$, while for nonvanishing $V_{k}$ and $h_{k}$ we utilize a neuroevolution algorithm [45] to design suitable quantum circuits (see Methods). With the obtained quantum circuits, we perform our experiment on a flip-chip superconducting quantum processor (Fig. 1e) with a chain of $L=14$ transmon qubits denoted as $Q_{1}$ through $Q_{L}$ (Fig. 1a). See the Method and Supplementary Information for the details of the experimental setup.

\section{Symmetry breaking at boundaries}

The characteristic signature of an FSPT phase is the breaking of the discrete time-translational symmetry at the boundaries of the chain but not in the bulk. This can be manifested by the persistent oscillation with period $2 T$ of local magnetizations at the boundaries. In Fig. 2, we plot the time evolution of disorder-averaged local magnetizations $\sigma_{j}^{z}(t)$ for different phases. From Fig. 2a, it is evident that in the FSPT phase, the disorder-averaged magnetizations at the two ends of the chain, namely $\sigma_{1}^{z}(t)$ and $\sigma_{L}^{z}(t)$, oscillate with a $2 T$ periodicity, for up to 20 driving cycles. In stark contrast, the local magnetizations in the bulk of the chain $\left(\sigma_{j}^{z}(t)\right.$ with $\left.2 \leq j \leq L-1\right)$ decay quickly to zero and do not show period-doubled oscillations. This unconventional behavior is independent of disorder averaging. Even for a single random disorder instance, the magnetizations exhibit similar dynamical features, as shown in Fig. 2b. The distinction between the dynamics of boundary and bulk magnetizations can also be clearly seen by examining $\sigma_{j}^{z}(t)$ in the frequency domain. As shown in Fig. 2d, the edge spins lock to the subharmonic frequency of the drive pe$\operatorname{riod} \omega / \omega_{0}=1 / 2$ whereas the bulk spins show no such peak. We stress that the subharmonic response for the edge spins obtained in our experiment is notably robust to various perturbations and experimental imperfections (see Supplementary Information I. B for a more in-depth discussion). For comparison, we also experimentally measure the dynamics of the magnetizations in the thermal phase. Our results are shown in Fig. 2c and Fig. 2e, where we see that the magnetizations for both the edge and bulk spins decay quickly to zero and no subharmonic response appears at all.

The breaking of the discrete time translational symmetry at the boundaries can also be detected by the disorder-averaged autocorrelators defined as $\bar{A}_{j}=\overline{\left\langle\Psi_{0}\left|\sigma_{j}^{z}(t) \sigma_{j}^{z}(0)\right| \Psi_{0}\right\rangle}$. Our experimental measurements of autocorrelators for up to 40 driving cycles are plotted in Fig. 2f, again showing the breaking of time translational symmetry at the boundaries but not in the bulk. We mention that, in the FSPT phase, the local magnetizations for the edge spins exhibit a gradually decaying envelope, which could be attributed to either external circuit errors or slow internal thermalization. To distinguish these two mechanisms, we carry out an additional experiment on the "echo" circuit $U_{\text {echo }} \equiv\left(U_{F}^{\dagger}\right)^{t} U_{F}^{t}$, whose deviation from the identity operator measures the effect of circuit errors [18]. The square root of the output of $U_{\text {echo }}$ (black solid lines shown in Fig. 2f) fits well with the decaying envelope of the results obtained by evolution under $U_{F}$. This indicates that the decay of the envelope is due to circuit errors rather than thermalization, which corroborates that the system is indeed in the localized phase.

\section{Localization-protected topological states}

In the above discussion, the initial states are random product states. To establish the FSPT phase, additional experiments on other initial states and other local observables are necessary. In this section, we show that the stabilizers in the bulk do not break the discrete time translational symmetry, but at the boundaries they do. To understand this, we consider the idealized cluster-state and spin-flip limit, i.e., $V_{k}=h_{k}=0$ and $\delta=0$. In this limit, $H_{2}$ reduces to a summation of stabilizers: $H_{2}=-\sum_{k=2}^{L-1} J_{k} S_{k}$ with $S_{k} \equiv \hat{\sigma}_{k-1}^{z} \hat{\sigma}_{k}^{x} \hat{\sigma}_{k+1}^{z}$. To break the degeneracy of $\mathrm{H}_{2}$, we consider adding two bound- 


$$
\text { c }
$$

a
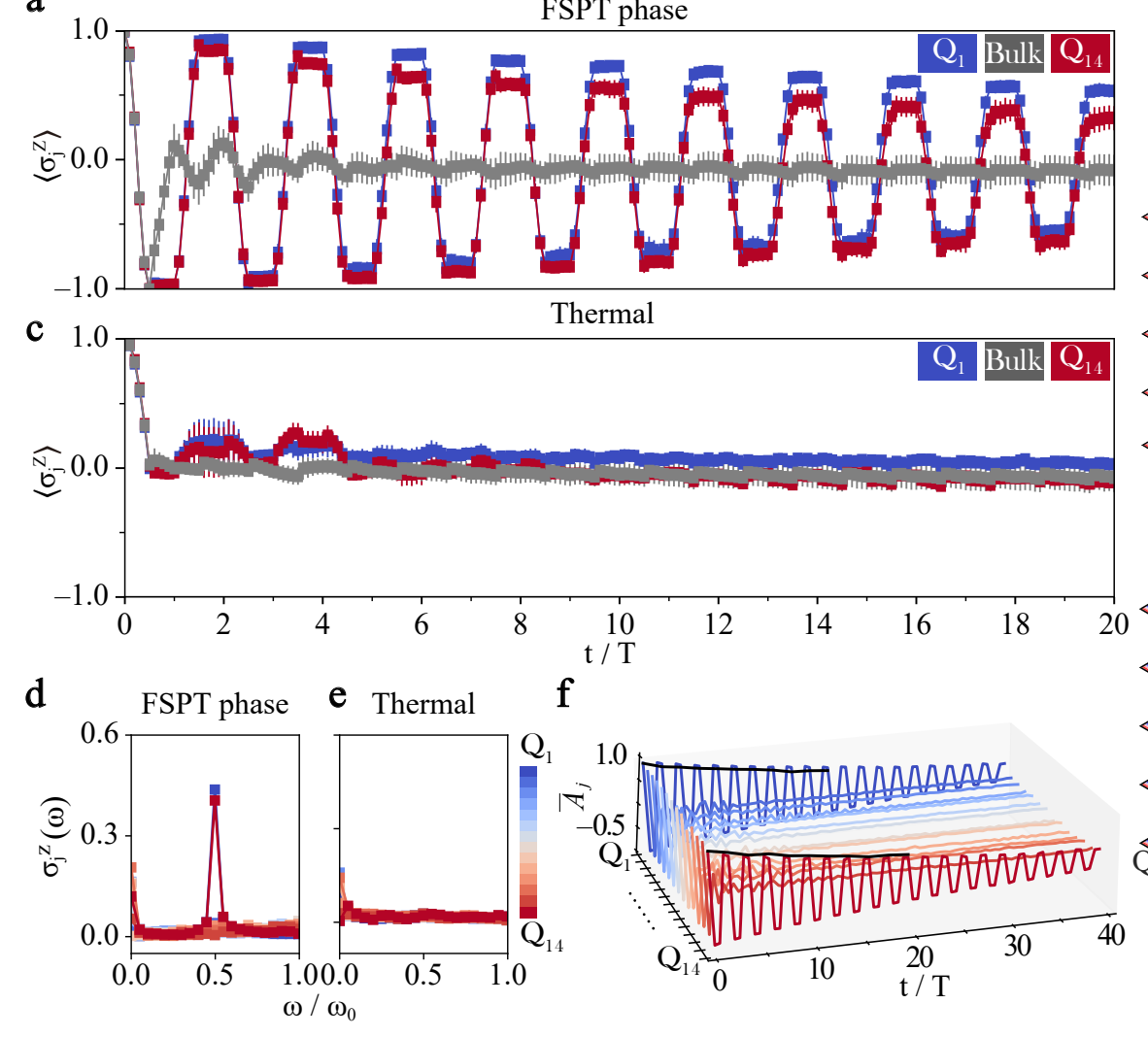

FSPT phase

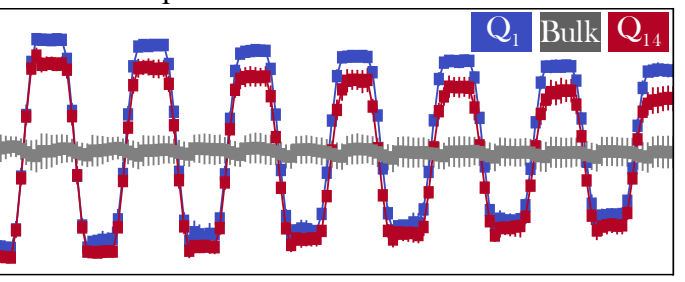

b 1.0 0.0 $-1.0\left\langle\sigma_{j}^{\mathrm{Z}}\right\rangle$

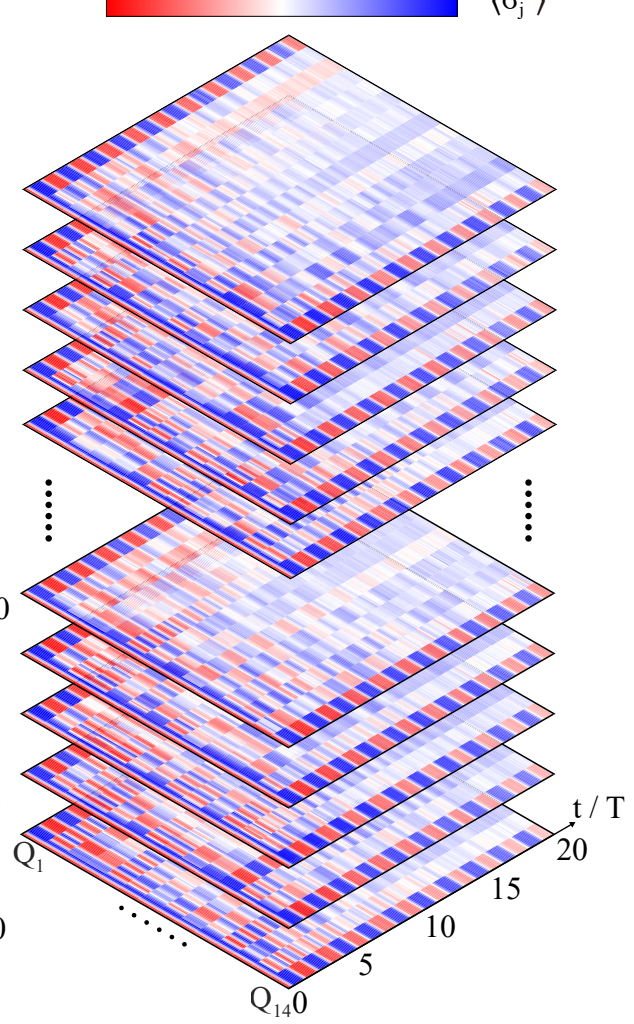

FIG. 2. Observation of an FSPT phase. a, Time evolution of disorder-averaged local magnetizations deep in the FSPT phase $\left(J=\Delta_{J}=1\right.$, $V=h=\Delta_{V}=\Delta_{h}=0$, and $\delta=0.01$ ). The initial states are $|0\rangle^{\otimes L}$ and data shown is averaged over 20 random disorder instances. While the bulk magnetization decays quickly to zero, the edge spins oscillate with a stable subharmonic response for up to 20 cycles. $\mathbf{b}$, The evolution dynamics of local magnetizations for different random instances. Here, each layer corresponds to a specific random instance. c, Magnetization dynamics deep in the thermal phase $\left(J=\Delta_{J}=1, V=h=\Delta_{V}=\Delta_{h}=0\right.$, and $\left.\delta=0.8\right)$. d, Fourier transform of experimentally measured $\left\langle\sigma_{j}^{z}(t)\right\rangle$ in the FSPT phase. The edge spins lock to the subharmonic frequency, which is in sharp contrast to the bulk spins. e, Fourier spectra of $\left\langle\sigma_{j}^{z}(t)\right\rangle$ in the thermal phase. No robust subharmonic frequency peak appears for either edge spins or bulk spins in this case. f. Time-dependence of the autocorrelator $\bar{A}_{j}=\overline{\left\langle\Psi_{0}\left|\sigma_{j}^{z}(t) \sigma_{j}^{z}(0)\right| \Psi_{0}\right\rangle}$ for up to 40 cycles, obtained from averaging over 20 random instances deep in the FSPT phase, with the initial states prepared as random product states in the computational basis. The black solid lines show the results of "echo" circuits for two boundary qubits.

ary terms $J_{1} S_{1}\left(S_{1} \equiv \hat{\sigma}_{1}^{x} \hat{\sigma}_{2}^{z}\right)$ and $J_{L} S_{L}\left(S_{L} \equiv \hat{\sigma}_{L}^{x} \hat{\sigma}_{L-1}^{z}\right)$, which are commuting with all bulk stabilizers, to the Hamiltonian $\mathrm{H}_{2}$, forming a new cluster-state Hamiltonian $\mathrm{H}_{2}^{\prime}=$ $H_{2}+J_{1} S_{1}+J_{L} S_{L}$. We note that the eigenstates of $H_{2}^{\prime}$ are also eigenstates of $H_{2}$ with degeneracy split by the boundary terms. We choose the initial states to be random eigenstates of $H_{2}^{\prime}$ and evolve the system with the time-periodic Hamiltonian $H(t)$ to measure the time-dependence of local stabilizers.

In Fig. 3a, we show a sketch of the quantum circuit used in our experiment to prepare the desired random eigenstates of $H_{2}^{\prime}$. To manifest the topological nature of these eigenstates, we study their entanglement spectra [50], which are widely used as a crucial diagnostic for universal topological properties of quantum phases [50-53]. In our experiment, we prepare random eigenstates of $\mathrm{H}_{2}$ with both open and periodic boundary conditions and obtain the reduced density matrices of half of the system, $\rho_{\text {half }}$, through quantum state tomography. Figure $3 \mathbf{b}$ displays the entanglement spectra $(\{-\ln v\}$ where $\{v\}$ are the eigenvalues of $\rho_{\text {half }}$ ) of two experimentally prepared eigenstates for open and periodic boundary conditions, respectively. From this figure, a clear two-fold degeneracy for the low-lying Schmidt states is obtained for the open boundary conditions. This degeneracy corresponds to an effectively decoupled spin-half degree of freedom at the boundary of the bipartition. For periodic boundary conditions, the spectrum is four-fold degenerate, corresponding to two effectively decoupled spins at the two boundaries of the bipartition. The degeneracy of the entanglement spectrum and its dependence on boundary conditions marks a characteristic feature of the SPT state prepared in our experiment. We remark that the degeneracy disappears above the entanglement gap. This is due to finite size effects and experimental imperfections.

In Fig. 3c, we plot the time-dependence of local stabilizers in the FSPT phase. We observe that the stabilizers at the boundaries oscillate with a $2 T$ periodicity, indicating again the breaking of discrete time translational symmetry at the bound- 
a

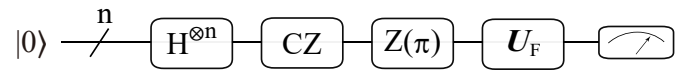

b

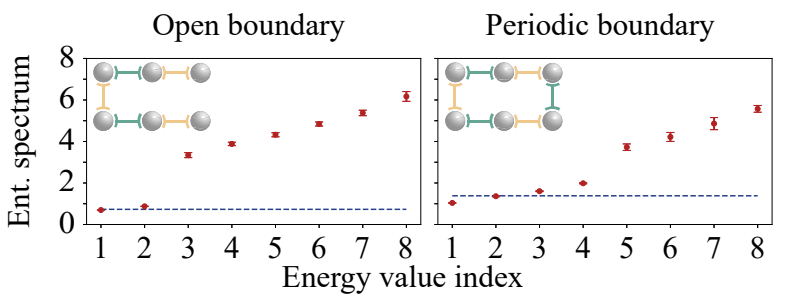

c

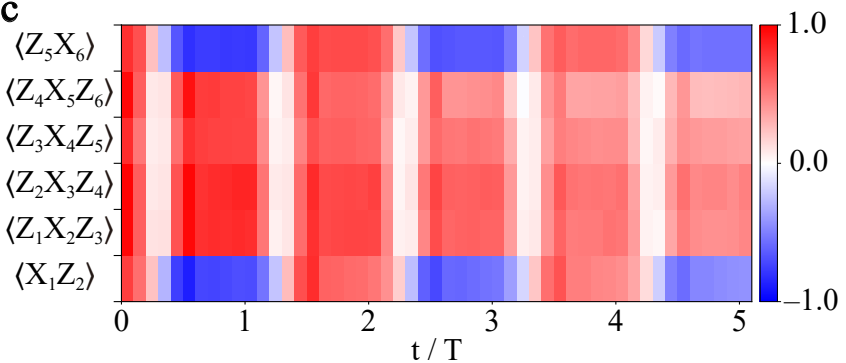

FIG. 3. Dynamics of stabilizers with random initial SPT states. a, Schematic of the experimental circuit for preparing random SPT states. To prepare the system into the ground state of the cluster Hamiltonian $H_{2}^{\prime}$, we apply a Hadamard gate $(\mathrm{H})$ on each qubit and then run $\mathrm{CZ}$ gates in parallel for all neighboring qubit pairs in two steps. Then we apply $\mathrm{Z}(\pi)$ on some random sites to create excitations and transfer the ground state to a highly excited eigenstate of $H_{2}^{\prime}$. This procedure enables the preparation of random SPT states at high energy. We then evolve these states with the Hamiltonian $H(t)$ to study the dynamics of stabilizers. b, Entanglement (abbreviated as "Ent.") spectra of random SPT states prepared in our experiment, with both open and periodic boundary conditions. The dashed lines indicate the lowest degenerate entanglement energies obtained by simulation in error-free case. The two- and four-fold degeneracy (in the case of open and periodic boundary conditions, respectively) of the low-lying entanglement levels is a characteristic feature of the topological nature of these states. c, The time dependence of stabilizers in the FSPT phase, averaged over 20 random circuit instances. The parameters in $\mathbf{b}$ and $\mathbf{c}$ are chosen as $L=6$, $\delta=0.1, J=\Delta_{J}=1, h=\Delta_{h}=0.01$, and $V=\Delta_{V}=0.01$.

aries. In the bulk, the stabilizers oscillate with a $T$ periodicity and are synchronized with the driving frequency, showing that no symmetry breaking occurs. This is in sharp contrast to the dynamics of bulk magnetizations, which decay rapidly to zero and exhibit no oscillation. In fact, in the FSPT phase, the system is many-body-localized and there exist a set of local integrals of motion, which are the "dressed" versions of the stabilizers with exponentially small tails [34]. The persistent oscillations of the bulk stabilizers observed in our experiment originate from these local integrals of motion and are a reflection of the fact that the system is indeed in an MBL phase.

\section{Phase transition}

We now turn to the phase transition between the FSPT phase and the trivial thermal phase. For simplicity and concreteness, we fix other parameters and vary the drive perturbation

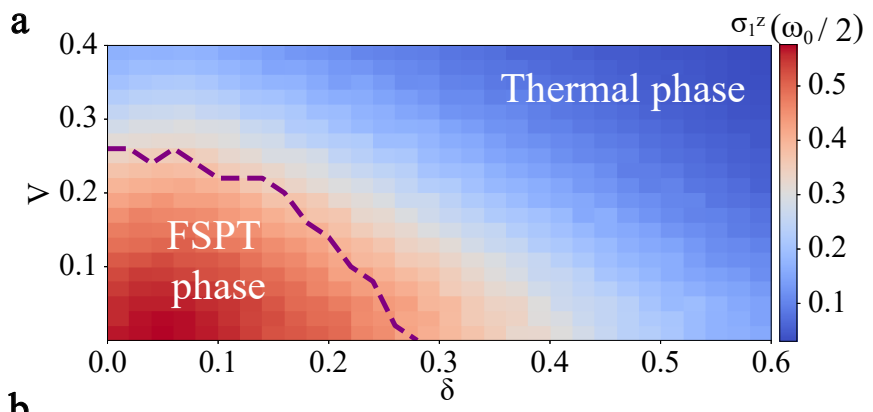

b

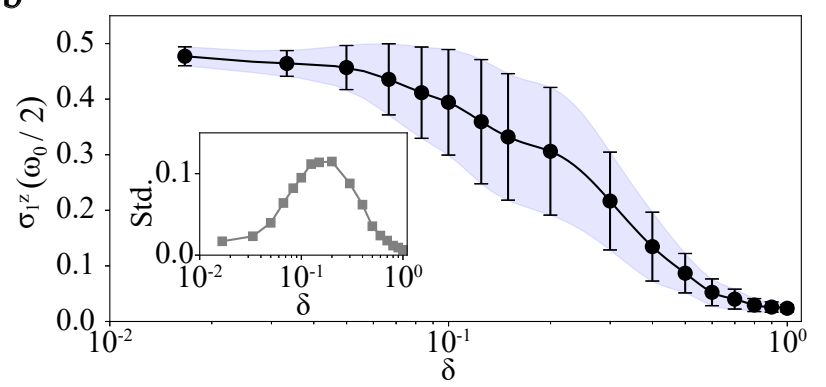

FIG. 4. Phase diagram and detection of phase transition. a, The numerical $\delta-V$ phase diagram obtained by examining the central subharmonic peak height for the edge spins in the Fourier spectrum, averaged over 1000 disorder instances. The dashed line corresponds to the maximal height variances for varying $V$ with each fixed $\delta$ point, which gives a rough estimation of the phase boundary. Here, the parameters are chosen as $L=8, J=\Delta_{J}=1$, and $h=\Delta_{h}=\Delta_{V}=0.05$. b. Experimental result of the subharmonic peak height as a function of $\delta$ with fixed $h=V=\Delta_{h}=\Delta_{V}=0$ and $J=\Delta_{J}=1$, averaged over 20 disorder instances uniformly sampling from the interval $\left[J-\Delta_{J}, J+\Delta_{J}\right]$ for 14 qubits, with the shadow outlining the standard deviation. Inset, the standard deviation of the central peak height as a function of $\delta$.

$\delta$ and the interaction strength $V$. Theoretically, the system is expected to exhibit an FSPT phase for small $\delta$ and $V$. With increasing $\delta$ and $V$, the strong interaction diminishes localization and eventually thermalizes the system. At some critical values of $\delta$ and $V$, a transition between these two phases occurs. In Fig. $4 \mathbf{a}$, we plot the $\delta-V$ phase diagram obtained from numerical simulations, where the phase boundary, although not very sharp due to finite-size effects, can be located and visualized approximately.

To experimentally examine this phase transition, we further fix the interaction strength $V=0$. We probe the transition point by measuring the variance of the subharmonic spectral peak height, i.e., the amplitude of the Fourier spectrum of $\left\langle\sigma_{1}^{z}\right\rangle$ at $\omega=\omega_{0} / 2$ for the boundary spin. Figure $4 \mathbf{b}$ shows the subharmonic peak height as a function of the drive perturbation $\delta$. At small $\delta$, the system is in the FSPT phase, and the peak height remains at a value around 0.5 . As we increase $\delta$ to a large value, the system transitions out of the topological phase and the peak height vanishes. This is consistent with the theoretical analysis above. The largest variance of the peak height corresponds to the phase transition point. The inset of Fig. $4 \mathbf{b}$ shows the measured standard deviation as a function of $\delta$, in- 
dicating a phase transition point around $\delta \approx 0.2$.

\section{Conclusion and outlook}

In summary, we have experimentally observed signatures of an intrinsically non-equilibrium Floquet SPT phase with a programmable superconducting quantum processor. In contrast to previously reported conventional time crystals, for our observed FSPT phase, the discrete time translational symmetry only breaks at the boundaries but not in the bulk. We measured the persistent oscillations of edge spins with a subharmonic frequency and experimentally demonstrated that the FSPT phase is robust to symmetry-respecting perturbations in the drive and imperfections in the experiment. In addition, we also demonstrated that the subharmonic response of boundary observables is independent of the initial state.

The controllability and scalability of the superconducting platform demonstrated in our experiment open up several new avenues for future fundamental studies and potential applications. In particular, it would be interesting and important to explore other exotic non-equilibrium phases beyond classical simulability [54], with superconducting or other near-term quantum platforms. In practice, the observed FSPT phase may have applications in some quantum information processing tasks, such as quantum metrology or implementing a robust quantum memory with topological protection.

\section{Methods}

\section{Characterization of the model Hamiltonian}

To understand why time translational symmetry breaks at the boundary but not in the bulk, we consider the idealized "cluster-model" limit ( $\left.V_{k}=h_{k}=0\right)$ and set $\delta=0$. We suppose that the system is initially prepared in a random product state in the computational basis, and we use the dynamics of local magnetization as a diagnostic. In this simple scenario, the topologically non-trivial structure of the cluster states (eigenstates of $U_{2}$ ) gives rise to edge modes that behave as free spins. At each driving period, the unitary operator $U_{1}$ flips all spins. As a result, the edge spins are reversed after one period and return to their initial configuration after two, leading to the period-doubled dynamics of the local magnetization at the boundaries. For spins in the bulk, however, the unitary operator $U_{2}$ plays a role and evolves the random product state to a state with vanishing magnetization, resulting in no period doubling. When $V_{k}=0$, the Hamiltonian (1) can be mapped to free Majorana fermions (see Supplementary Information I. B and, e.g., Refs. [55, 56]). Further setting $\delta=h_{k}=0$, we find that Eq. (1) maps onto two decoupled copies of the fixedpoint model of a $\mathbb{Z}_{2}$ FSPT phase considered in Ref. [39]. The robustness of the subharmonic responses of the topologically protected edge spins to perturbations respecting the $\mathbb{Z}_{2} \times \mathbb{Z}_{2}$ symmetry is discussed in depth in the Supplementary Information I. B.

\section{Logarithmic entanglement growth}

For a many-body localized system, the entanglement entropy will feature a logarithmic growth [57], which is in sharp contrast to the case of Anderson localization without interactions. For the model Hamiltonian $H(t)$ studied in this paper, we also expect a logarithmic growth of the entanglement entropy inside the FSPT phase with $V_{k} \neq 0$. We numerically simulate the entanglement dynamics of the system deep in the FSPT phase with the time-evolving block decimation algorithm up to a system size $L=100$ (see Supplementary Information II.). Our results clearly verify the logarithmic entanglement growth, which again implies that the FSPT phase in indeed many-body localized with nonvanishing $V_{k}$. In our experiment, we also study the entanglement dynamics for a small system size through quantum tomography (see Supplementary Information IV.). We find that in the thermal phase, the entanglement grows much faster than that for the FSPT phase. However, due to decoherence and other experimental imperfections, we are not able to observe the logarithmic entanglement growth.

\section{Quantum circuits for implementing $H(t)$}

Direct implementation of the Floquet Hamiltonian $H(t)$ with superconducting qubits faces a notable difficulty: the natural interactions hosted by the superconducting qubits are only two-body, so the three-body terms in $H_{2}$ cannot emerge directly. Fortunately, programmable superconducting qubits are universal for quantum computation; thus we can explore the idea of digital quantum simulation to emulate the dynamics of $H(t)$. However, due to inevitable experimental imperfections, the depth of the quantum circuits is limited. As a result, obtaining well-performing circuits with an optimal depth which can implement $H(t)$ (or equivalently the Floquet unitary $U_{F}$ ) is of crucial importance for the success of our experiment.

To find the desired quantum circuits, we utilize a neuroevolution method introduced in Ref. [45] which outputs a nearoptimal architecture for a family of variational quantum circuits that can implement $H(t)$ with different random disorder instances. For a given instance of $J_{k}, V_{k}$, and $h_{k}$, we use the gradient decent method to tune the variational parameters of the ansatz circuits to minimize the distance between the unitary represented by the circuit and the unitary generated by $H(t)$ within a samll time interval. In the idealized "clustermodel" limit ( $V_{k}=h_{k}=0$ ), we can find a simple exact oneto-one correspondence between $J_{k}$ and the variational parameters, independent of the system size and the values of $J_{k}$ and $\delta$. Thus, we are able to construct an analytical quantum circuit that can implement $H(t)$ precisely, and at the same time are experimentally friendly and practical. The details of how to obtain the desired quantum circuits are given in Sec. III of the Supplementary Information.

\section{Experimental setup}

Our experiment is performed on a flip-chip superconducting quantum processor designed to encapsulate a square array of $6 \times 6$ transmon qubits with adjustable nearest-neighbor couplings (Fig. 1e), on which a chain of up to $L=14$ qubits, denoted as $Q_{1}$ through $Q_{L}$, that alternate with $L-1$ cou- 
plers, denoted as $C_{1}$ through $C_{L-1}$, are selected to observe the FSPT phase (Fig. 1a). All $L$ qubits can be individually tuned in frequency with flux biases, excited by microwaves, and measured using on-chip readout resonators; all couplers are also of transmon type with characteristic transition frequencies higher than those of the qubits, which can be controlled with flux biases to tune the effective nearest-neighbor couplings. During an experimental sequence (Fig. 1d), we first initialize each qubit, $Q_{j}$, in $|0\rangle$ at its idle frequency $\omega_{j}$, following which we alternate the single-qubit gates at $\omega_{j}$ with the two-qubit controlled- $\pi(\mathrm{CZ})$ gates realized by biasing $Q_{j}$ and its neighboring qubit to the pairwise frequencies listed in $\left(\omega_{j}^{\mathrm{A}(\mathrm{B})}, \omega_{j+1}^{\mathrm{A}(\mathrm{B})}\right)$ for a fixed interaction time (see Supplementary Information III. C). Meanwhile, each coupler is dynamically switched between two frequencies [58-63]: one is to turn off the effective coupling where the neighboring two qubits can be initialized and operated with single-qubit gates; the other one is to turn on the nearest-neighbor coupling to around $11 \mathrm{MHz}$ for a $\mathrm{CZ}$ gate. After $n$ layers of the alternating single- and two-qubit gates, we finally tune all qubits to their respective $\omega_{j}^{\mathrm{m}}$ for simultaneous quantum-state measurement. Qubit energy relaxation times measured around $\omega_{j}$ are in the range of 11 to $37 \mu \mathrm{s}$. More characteristic qubit parameters, including the above mentioned frequencies, anharmonicities, and readout fidelities, can be found in Supplementary Information Tab. S1.

We explore a quantum digital simulation scheme to implement the dynamics of the system under the driven Hamiltonian $H(t)$. More specifically, we decompose the evolution operators into the experimentally feasible single-qubit gates $[\mathrm{X}(\theta), \mathrm{Y}(\theta)$, and $\mathrm{Z}(\theta)]$ and two-qubit gates $\left[\mathrm{CR}_{z}( \pm \pi)\right]$, where $\mathrm{X}(\theta), \mathrm{Y}(\theta)$, and $\mathrm{Z}(\theta)$ are rotations around $x-, y-$, and $z$ axis by the angle $\theta$, respectively, and $\mathrm{CR}_{z}( \pm \pi)$ are the $z$-axis rotations of the target qubit by $\pm \pi$ conditioned on the state of the control qubit (see Fig. 1d and Supplementary Information III. A for the ansatz that generates the gate sequences). $\mathrm{X}(\theta)$ and $\mathrm{Y}(\theta)$ are realized by applying $50 \mathrm{~ns}$-long microwave pulses with full width half maximum of $25 \mathrm{~ns}$, whose quadrature correction terms are optimized to minimize state leakages to higher levels [64]. Simultaneous randomized benchmarkings indicate that the single-qubit gates used in this experiment have reasonably high fidelities, averaging above 0.99 (see Supplementary Information Tab. $S 1) . Z(\theta)$ is realized using the virtual- $Z$ gate, which encodes the information $\theta$ in the rotation axes of all subsequent gates [65], and is combined with $\mathrm{CZ}$ to assemble $\mathrm{CR}_{z}( \pm \pi)$. Here, we adopt the strategy reported elsewhere $[62,66]$ to realize the $\mathrm{CZ}$ gate, i.e., we diabatically tune the coupler frequency while keeping $|11\rangle$ and $|02\rangle$ (or $|20\rangle$ ) for the subspace of the two neighboring qubits in near resonance. The 40 ns-long CZ gate for a pair of neighboring qubits can be individually optimized to be around 0.99 in fidelity as calibrated by interleaved randomized benchmarking; when simultaneously running the $\mathrm{CZ}$ gates for multiple pairs of neighboring qubits as required in the experimental sequence, the averaged $\mathrm{CZ}$ gate fidelities can be around 0.985 as obtained by simultaneous randomized benchmarking (see Supplementary Information Tab. S1).

Data availability The data presented in the figures and that support the other findings of this study are available upon reasonable request from the corresponding authors.

Code availability The data analysis and numerical simulation codes are available from the corresponding authors on reasonable request.

Acknowledgement We thank L.M. Duan and S.L. Xu for helpful discussion. The device was fabricated at the MicroNano Fabrication Center of Zhejiang University. X.Zha., J.D., K.W., J.C., P.Z., W.R., H.D., S.X., Y.G., F.J., X.Zhu., Q.G., H.L., C.S., Z.W. and H.W. acknowledge the support of the National Natural Science Foundation of China (Grants No. 11725419, U20A2076, and 92065204), the National Basic Research Program of China (Grants No. 2017YFA0304300), the Zhejiang Province Key Research and Development Program (Grant No. 2020C01019), and the Key-Area Research and Development Program of Guangdong Province (Grant No. 2020B0303030001). W.J. and D.-L.D. are supported by the National Natural Science Foundation of China (Grants No. 12075128), Tsinghua University, and the Shanghai Qi Zhi Institute. A.V.G. and F.L. acknowledge funding by the AFOSR.

Author contributions X.Zha. and J.D. carried out the experiments under the supervision of Z.W. and H.W.. H.L., Z.W., and J.C. fabricated the device supervised by H.W.. W.J. performed the numerical simulations. A.V.G., T.I., F.L., Z.-X.G., and D.-L.D. conducted the theoretical analysis. All authors contributed to the experimental set-up, the discussions of the results and the writing of the manuscript.

Competing interests All authors declare no competing interests.

\footnotetext{
* These authors contributed equally to this work.

† 2010wangzhen@zju.edu.cn

$\ddagger$ dldeng@tsinghua.edu.cn
}

[1] F. Wilczek, Quantum time crystals, Phys. Rev. Lett. 109, 160401 (2012).

[2] D. V. Else, B. Bauer, and C. Nayak, Floquet time crystals, Phys. Rev. Lett. 117, 090402 (2016).

[3] N. Y. Yao, A. C. Potter, I.-D. Potirniche, and A. Vishwanath, Discrete time crystals: Rigidity, criticality, and realizations, Phys. Rev. Lett. 118, 030401 (2017).

[4] V. Khemani, A. Lazarides, R. Moessner, and S. L. Sondhi, Phase structure of driven quantum systems, Phys. Rev. Lett. 116, 250401 (2016).

[5] K. Sacha and J. Zakrzewski, Time crystals: a review, arXiv:1704.03735 (2017). 
[6] D. V. Else, C. Monroe, C. Nayak, and N. Y. Yao, Discrete time crystals, Annu. Rev. Condens. Matter Phys. 11, 467 (2020).

[7] N. Y. Yao and C. Nayak, Time crystals in periodically driven systems, Physics Today 71, 40 (2018).

[8] V. Khemani, R. Moessner, and S. Sondhi, A brief history of time crystals, arXiv:1910.10745 (2019).

[9] J. Zhang, P. Hess, A. Kyprianidis, P. Becker, A. Lee, J. Smith, G. Pagano, I.-D. Potirniche, A. Potter, A. Vishwanath, et al., Observation of a discrete time crystal, Nature 543, 217 (2017).

[10] A. Kyprianidis, F. Machado, W. Morong, P. Becker, K. S. Collins, D. V. Else, L. Feng, P. W. Hess, C. Nayak, G. Pagano, et al., Observation of a prethermal discrete time crystal, Science 372, 1192 (2021).

[11] S. Choi, J. Choi, R. Landig, G. Kucsko, H. Zhou, J. Isoya, F. Jelezko, S. Onoda, H. Sumiya, V. Khemani, et al., Observation of discrete time-crystalline order in a disordered dipolar many-body system, Nature 543, 221 (2017).

[12] J. O'Sullivan, O. Lunt, C. W. Zollitsch, M. Thewalt, J. J. Morton, and A. Pal, Signatures of discrete time crystalline order in dissipative spin ensembles, New Journal of Physics 22, 085001 (2020).

[13] J. Randall, C. Bradley, F. van der Gronden, A. Galicia, M. Abobeih, M. Markham, D. Twitchen, F. Machado, N. Yao, and T. Taminiau, Observation of a many-body-localized discrete time crystal with a programmable spin-based quantum simulator, arXiv:2107.00736 (2021).

[14] J. Smits, L. Liao, H. T. C. Stoof, and P. van der Straten, Observation of a space-time crystal in a superfluid quantum gas, Phys. Rev. Lett. 121, 185301 (2018).

[15] S. Autti, V. B. Eltsov, and G. E. Volovik, Observation of a time quasicrystal and its transition to a superfluid time crystal, Phys. Rev. Lett. 120, 215301 (2018).

[16] J. Rovny, R. L. Blum, and S. E. Barrett, Observation of discretetime-crystal signatures in an ordered dipolar many-body system, Phys. Rev. Lett. 120, 180603 (2018).

[17] S. Pal, N. Nishad, T. S. Mahesh, and G. J. Sreejith, Temporal order in periodically driven spins in star-shaped clusters, Phys. Rev. Lett. 120, 180602 (2018).

[18] X. Mi, M. Ippoliti, C. Quintana, A. Greene, Z. Chen, J. Gross, F. Arute, K. Arya, J. Atalaya, R. Babbush, et al., Observation of time-crystalline eigenstate order on a quantum processor, arXiv:2107.13571 (2021).

[19] C. Ying, Q. Guo, S. Li, M. Gong, X.-H. Deng, F. Chen, C. Zha, Y. Ye, C. Wang, Q. Zhu, et al., Floquet prethermal phase protected by u (1) symmetry on a superconducting quantum processor, arXiv:2107.07311 (2021).

[20] H. Xu, J. Zhang, J. Han, Z. Li, G. Xue, W. Liu, Y. Jin, and $\mathrm{H}$. Yu, Realizing discrete time crystal in an one-dimensional superconducting qubit chain, arXiv:2108.00942 (2021).

[21] J. Preskill, Quantum computing in the NISQ era and beyond, Quantum 2, 79 (2018).

[22] F. Pollmann, E. Berg, A. M. Turner, and M. Oshikawa, Symmetry protection of topological phases in one-dimensional quantum spin systems, Phys. Rev. B 85, 075125 (2012).

[23] X. Chen, Z.-C. Gu, Z.-X. Liu, and X.-G. Wen, Symmetryprotected topological orders in interacting bosonic systems, Science 338, 1604 (2012).

[24] X. Chen, Z.-C. Gu, Z.-X. Liu, and X.-G. Wen, Symmetry protected topological orders and the group cohomology of their symmetry group, Phys. Rev. B 87, 155114 (2013).

[25] T. Senthil, Symmetry-protected topological phases of quantum matter, Annu. Rev. Condens. Matter Phys. 6, 299 (2015).

[26] C.-K. Chiu, J. C. Y. Teo, A. P. Schnyder, and S. Ryu, Classification of topological quantum matter with symmetries, Rev. Mod.
Phys. 88, 035005 (2016).

[27] R. Nandkishore and D. A. Huse, Many-body localization and thermalization in quantum statistical mechanics, Annu. Rev. Condens. Matter Phys. 6, 15 (2015).

[28] M. Schreiber, S. S. Hodgman, P. Bordia, H. P. Lüschen, M. H. Fischer, R. Vosk, E. Altman, U. Schneider, and I. Bloch, Observation of many-body localization of interacting fermions in a quasi-random optical lattice, Science 349, 842 (2015).

[29] J. Smith, A. Lee, P. Richerme, B. Neyenhuis, P. W. Hess, P. Hauke, M. Heyl, D. A. Huse, and C. Monroe, Many-body localization in a quantum simulator with programmable random disorder, Nat. Phys. 10.1038/nphys3783 (2016).

[30] K. Xu, J.-J. Chen, Y. Zeng, Y.-R. Zhang, C. Song, W. Liu, Q. Guo, P. Zhang, D. Xu, H. Deng, K. Huang, H. Wang, X. Zhu, D. Zheng, and H. Fan, Emulating many-body localization with a superconducting quantum processor, Phys. Rev. Lett. 120, 050507 (2018).

[31] D. A. Abanin, E. Altman, I. Bloch, and M. Serbyn, Colloquium: Many-body localization, thermalization, and entanglement, Rev. Mod. Phys. 91, 021001 (2019).

[32] D. A. Huse, R. Nandkishore, V. Oganesyan, A. Pal, and S. L. Sondhi, Localization-protected quantum order, Phys. Rev. B 88, 014206 (2013).

[33] A. Chandran, V. Khemani, C. R. Laumann, and S. L. Sondhi, Many-body localization and symmetry-protected topological order, Phys. Rev. B 89, 144201 (2014).

[34] Y. Bahri, R. Vosk, E. Altman, and A. Vishwanath, Localization and topology protected quantum coherence at the edge of hot matter, Nat. Commun. 6, 7341 (2015).

[35] S. A. Parameswaran, A. C. Potter, and R. Vasseur, Eigenstate phase transitions and the emergence of universal dynamics in highly excited states, Ann. Phys. (Berl.) 529, 1600302 (2017).

[36] S. A. Parameswaran and R. Vasseur, Many-body localization, symmetry and topology, Rep. Prog. Phys. 81, 082501 (2018).

[37] P. Ponte, A. Chandran, Z. Papić, and D. A. Abanin, Periodically driven ergodic and many-body localized quantum systems, Ann. Phys. (N. Y.) 353, 196 (2015).

[38] F. Harper, R. Roy, M. S. Rudner, and S. Sondhi, Topology and broken symmetry in floquet systems, Annu. Rev. Condens. Matter Phys. 11, 345 (2020).

[39] C. W. von Keyserlingk and S. L. Sondhi, Phase structure of onedimensional interacting floquet systems. i. abelian symmetryprotected topological phases, Phys. Rev. B 93, 245145 (2016).

[40] D. V. Else and C. Nayak, Classification of topological phases in periodically driven interacting systems, Phys. Rev. B 93, 201103 (2016).

[41] A. C. Potter, T. Morimoto, and A. Vishwanath, Classification of interacting topological floquet phases in one dimension, Phys. Rev. X 6, 041001 (2016).

[42] R. Roy and F. Harper, Periodic table for floquet topological insulators, Phys. Rev. B 96, 155118 (2017).

[43] H. Watanabe and M. Oshikawa, Absence of quantum time crystals, Phys. Rev. Lett. 114, 251603 (2015).

[44] P. T. Dumitrescu, J. Bohnet, J. Gaebler, A. Hankin, D. Hayes, A. Kumar, B. Neyenhuis, R. Vasseur, and A. C. Potter, Realizing a dynamical topological phase in a trapped-ion quantum simulator, arXiv:2107.09676 (2021).

[45] Z. Lu, P.-X. Shen, and D.-L. Deng, Markovian Quantum Neuroevolution for Machine Learning, arXiv:2012.15131 (2020).

[46] C. W. von Keyserlingk, V. Khemani, and S. L. Sondhi, Absolute stability and spatiotemporal long-range order in floquet systems, Phys. Rev. B 94, 085112 (2016).

[47] V. Khemani, C. W. von Keyserlingk, and S. L. Sondhi, Defining time crystals via representation theory, Phys. Rev. B 96, 115127 
(2017).

[48] A. Kumar, P. T. Dumitrescu, and A. C. Potter, String order parameters for one-dimensional floquet symmetry protected topological phases, Phys. Rev. B 97, 224302 (2018).

[49] I. M. Georgescu, S. Ashhab, and F. Nori, Quantum simulation, Rev. Mod. Phys. 86, 153 (2014).

[50] H. Li and F. D. M. Haldane, Entanglement spectrum as a generalization of entanglement entropy: Identification of topological order in non-abelian fractional quantum hall effect states, Phys. Rev. Lett. 101, 010504 (2008).

[51] B. Swingle and T. Senthil, Geometric proof of the equality between entanglement and edge spectra, Phys. Rev. B 86, 045117 (2012).

[52] L. Fidkowski, Entanglement spectrum of topological insulators and superconductors, Phys. Rev. Lett. 104, 130502 (2010).

[53] V. Alba, M. Haque, and A. M. Läuchli, Boundary-locality and perturbative structure of entanglement spectra in gapped systems, Phys. Rev. Lett. 108, 227201 (2012).

[54] F. M. Surace, A. Russomanno, M. Dalmonte, A. Silva, R. Fazio, and F. Iemini, Floquet time crystals in clock models, Phys. Rev. B 99, 104303 (2019).

[55] P. Fendley, Parafermionic edge zero modes inzn-invariant spin chains, J. Stat. Mech.: Theory Exp. (11), P11020.

[56] T. Iadecola, L. H. Santos, and C. Chamon, Stroboscopic symmetry-protected topological phases, Phys. Rev. B 92, 125107 (2015).

[57] J. H. Bardarson, F. Pollmann, and J. E. Moore, Unbounded growth of entanglement in models of many-body localization, Phys. Rev. Lett. 109, 017202 (2012).

[58] F. Yan, P. Krantz, Y. Sung, M. Kjaergaard, D. L. Campbell, T. P. Orlando, S. Gustavsson, and W. D. Oliver, Tunable coupling scheme for implementing high-fidelity two-qubit gates, Phys. Rev. Applied 10, 054062 (2018).

[59] F. Arute, K. Arya, R. Babbush, D. Bacon, J. C. Bardin, R. Barends, R. Biswas, S. Boixo, F. G. S. L. Brandao, D. A. Buell, B. Burkett, Y. Chen, Z. Chen, B. Chiaro, R. Collins, W. Courtney, A. Dunsworth, E. Farhi, B. Foxen, A. Fowler, C. Gidney, M. Giustina, R. Graff, K. Guerin, S. Habegger, M. P. Harrigan, M. J. Hartmann, A. Ho, M. Hoffmann, T. Huang, T. S. Humble, S. V. Isakov, E. Jeffrey, Z. Jiang, D. Kafri, K. Kechedzhi, J. Kelly, P. V. Klimov, S. Knysh, A. Korotkov, F. Kostritsa, D. Landhuis, M. Lindmark, E. Lucero, D. Lyakh, S. MandrÃ , J. R. McClean, M. McEwen, A. Megrant, X. Mi, K. Michielsen, M. Mohseni, J. Mutus, O. Naaman, M. Neeley, C. Neill, M. Y. Niu, E. Ostby, A. Petukhov, J. C. Platt, C. Quintana, E. G. Rieffel, P. Roushan, N. C. Rubin, D. Sank, K. J. Satzinger, V. Smelyanskiy, K. J. Sung, M. D. Trevithick, A. Vainsencher, B. Villalonga, T. White, Z. J. Yao, P. Yeh, A. Zalcman, H. Neven, and J. M. Martinis, Quantum supremacy using a programmable superconducting processor, Nature $\mathbf{5 7 4}$,
505 (2019).

[60] Y. Xu, J. Chu, J. Yuan, J. Qiu, Y. Zhou, L. Zhang, X. Tan, Y. Yu, S. Liu, J. Li, F. Yan, and D. Yu, High-fidelity, high-scalability two-qubit gate scheme for superconducting qubits, Phys. Rev. Lett. 125, 240503 (2020).

[61] M. C. Collodo, J. Herrmann, N. Lacroix, C. K. Andersen, A. Remm, S. Lazar, J.-C. Besse, T. Walter, A. Wallraff, and C. Eichler, Implementation of conditional phase gates based on tunable $z z$ interactions, Phys. Rev. Lett. 125, 240502 (2020).

[62] Y. Sung, L. Ding, J. Braumüller, A. Vepsäläinen, B. Kannan, M. Kjaergaard, A. Greene, G. O. Samach, C. McNally, D. Kim, A. Melville, B. M. Niedzielski, M. E. Schwartz, J. L. Yoder, T. P. Orlando, S. Gustavsson, and W. D. Oliver, Realization of high-fidelity $\mathrm{cz}$ and $z z$-free iswap gates with a tunable coupler, Phys. Rev. X 11, 021058 (2021).

[63] Y. Wu, W.-S. Bao, S. Cao, F. Chen, M.-C. Chen, X. Chen, T.-H. Chung, H. Deng, Y. Du, D. Fan, M. Gong, C. Guo, C. Guo, S. Guo, L. Han, L. Hong, H.-L. Huang, Y.-H. Huo, L. Li, N. Li, S. Li, Y. Li, F. Liang, C. Lin, J. Lin, H. Qian, D. Qiao, H. Rong, H. Su, L. Sun, L. Wang, S. Wang, D. Wu, Y. Xu, K. Yan, W. Yang, Y. Yang, Y. Ye, J. Yin, C. Ying, J. Yu, C. Zha, C. Zhang, H. Zhang, K. Zhang, Y. Zhang, H. Zhao, Y. Zhao, L. Zhou, Q. Zhu, C.-Y. Lu, C.-Z. Peng, X. Zhu, and J.-W. Pan, Strong quantum computational advantage using a superconducting quantum processor, arXiv:2106.14734 (2021).

[64] C. Song, K. Xu, W. Liu, C.-p. Yang, S.-B. Zheng, H. Deng, Q. Xie, K. Huang, Q. Guo, L. Zhang, P. Zhang, D. Xu, D. Zheng, X. Zhu, H. Wang, Y.-A. Chen, C.-Y. Lu, S. Han, and J.-W. Pan, 10-qubit entanglement and parallel logic operations with a superconducting circuit, Phys. Rev. Lett. 119, 180511 (2017).

[65] D. C. McKay, C. J. Wood, S. Sheldon, J. M. Chow, and J. M. Gambetta, Efficient $z$ gates for quantum computing, Phys. Rev. A 96, 022330 (2017).

[66] B. Foxen, C. Neill, A. Dunsworth, P. Roushan, B. Chiaro, A. Megrant, J. Kelly, Z. Chen, K. Satzinger, R. Barends, F. Arute, K. Arya, R. Babbush, D. Bacon, J. C. Bardin, S. Boixo, D. Buell, B. Burkett, Y. Chen, R. Collins, E. Farhi, A. Fowler, C. Gidney, M. Giustina, R. Graff, M. Harrigan, T. Huang, S. V. Isakov, E. Jeffrey, Z. Jiang, D. Kafri, K. Kechedzhi, P. Klimov, A. Korotkov, F. Kostritsa, D. Landhuis, E. Lucero, J. McClean, M. McEwen, X. Mi, M. Mohseni, J. Y. Mutus, O. Naaman, M. Neeley, M. Niu, A. Petukhov, C. Quintana, N. Rubin, D. Sank, V. Smelyanskiy, A. Vainsencher, T. C. White, Z. Yao, P. Yeh, A. Zalcman, H. Neven, and J. M. Martinis (Google AI Quantum), Demonstrating a continuous set of two-qubit gates for near-term quantum algorithms, Phys. Rev. Lett. 125, 120504 (2020). 


\section{Supplementary Files}

This is a list of supplementary files associated with this preprint. Click to download.

- ObservationofFSPTPhaseSI.pdf 